\title{
Prognostic significance of multiple kallikreins in high-grade astrocytoma
}

\author{
Kristen L. Drucker ${ }^{1}$, Caterina Gianinni ${ }^{2}$, Paul A. Decker ${ }^{3}$, Eleftherios P. Diamandis ${ }^{4}$ and Isobel A. Scarisbrick ${ }^{1,5^{*}}$
}

\begin{abstract}
Background: Kallikreins have clinical value as prognostic markers in a subset of malignancies examined to date, including kallikrein 3 (prostate specific antigen) in prostate cancer. We previously demonstrated that kallikrein 6 is expressed at higher levels in grade IV compared to grade III astrocytoma and is associated with reduced survival of GBM patients.

Methods: In this study we determined KLK1, KLK6, KLK7, KLK8, KLK9 and KLK10 protein expression in two independent tissue microarrays containing 60 grade IV and 8 grade III astrocytoma samples. Scores for staining intensity, percent of tumor stained and immunoreactivity scores (IR, product of intensity and percent) were determined and analyzed for correlation with patient survival.

Results: Grade IV glioma was associated with higher levels of kallikrein-immunostaining compared to grade III specimens. Univariable Cox proportional hazards regression analysis demonstrated that elevated KLK6- or KLK7-IR was associated with poor patient prognosis. In addition, an increased percent of tumor immunoreactive for KLK6 or KLK9 was associated with decreased survival in grade IV patients. Kaplan-Meier survival analysis indicated that patients with KLK6-IR $<10$, KLK6 percent tumor core stained $<3$, or KLK7-IR $<9$ had a significantly improved survival. Multivariable analysis indicated that the significance of these parameters was maintained even after adjusting for gender and performance score.
\end{abstract}

Conclusions: These data suggest that elevations in glioblastoma KLK6, KLK7 and KLK9 protein have utility as prognostic markers of patient survival.

Keywords: Glioblastoma, Kallikrein, Prognosis

\section{Background}

Expression levels of select kallikreins (KLK) are proposed or already used as biomarkers in human malignancies, including prostate, ovarian or breast cancers. KLKs are a family of secreted serine proteases, consisting of 15 genes located in a contiguous cluster on chromosome 19q13.4 [1]. KLKs participate in trypsin- or chymotrypsin-like protein cleavage, leading to extracellular matrix degradation and tissue remodeling, activation or inactivation of other protease family members, or in some cases, activation of protease activated

\footnotetext{
* Correspondence: scarisbrick.isobel@mayo.edu

'Department of Physical Medicine and Rehabilitation, Mayo Medical and Graduate School, Mayo Clinic Rochester, 200 First Street SW, Rochester, MN 55905, USA

${ }^{5}$ Department of Physiology and Biomedical Engineering, Mayo Medical and Graduate School, Mayo Clinic Rochester, 200 First Street SW, Rochester, MN 55905, USA

Full list of author information is available at the end of the article
}

receptors (PARs) to elicit intracellular signaling and defined cellular responses [2]. For example, elevated levels of KLK6 are associated with higher grade, later stage and serous histotype ovarian cancer, all of which are associated with an unfavorable prognosis [3]. KLK3 (prostate specific antigen (PSA)) serves as a well-recognized serum biomarker for prostate cancer [4]. Providing the rationale for the current study, we recently demonstrated that elevated levels of KLK6 are associated with high-grade glioma and poor patient survival [5]. Very little is known regarding the potential prognostic significance of other kallikrein family members in glial tumors and here we examined the association of 6 kallikreins with GBM grade and patient survival.

The location of kallikrein family members on human chromosome 19q makes them of particular interest in glioma, given the frequency of copy number variations 
in glioma patient tumors [6, 7]. Whole arm loss of chromosome 19q has been linked to better survival in oligodendroglioma, although smaller deletions have not been shown to have the same survival benefit $[8,9]$. Conversely, gain of chromosome 19 in glioblastoma has been correlated with a poor prognosis [10], an effect attributed to radiation resistance [11]. In this regard it is of interest that not only are levels of KLK6 significantly elevated in high-grade glioma (glioblastoma multiforme (GBM), grade IV astrocytoma) and associated with poor patient survival, but in addition KLK6 promotes the resistance of glioma cells to a wide variety of cell deathinducing agents, including staurosporine, cisplatin, radiation and temozolomide [5]. The potential pathophysiological significance of KLK6 to glioma appears to extend to lower grade tumors as well, since patients with mixed intracranial tumors positive for KLK6 expression also have unfavorable prognoses compared to those lacking expression [12]. Interestingly, patients with KLK7 positive tumors also survived for shorter intervals post-surgery relative to patients in which no KLK7 expression was detected [13]. By contrast, tumor KLK8 RNA expression in the same patient cohort was not associated with survival [13].

Given the established prognostic significance of KLK6 to GBM patient survival, taken with the association of other KLKs with a variety of CNS tumor types, we made a comprehensive examination of five additional kallikreins in the patient cohort previously utilized to determine the prognostic significance of KLK6. Like KLK6, higher levels of KLK1, KLK7, KLK8, KLK9 and KLK10 were all found to be associated with higher astrocytoma grade. In addition, high tumor levels of KLK7-IR, were, like KLK6, found to be associated with reduced patient survival. These findings suggest that multiple kallikreins are positioned to play roles in the pathophysiology of high-grade glioma and that future studies are needed to determine their biological actions including roles in directing therapeutic response.

\section{Methods}

\section{Clinical samples}

The tissue microarrays containing grade III and grade IV astrocytoma specimens utilized in this study were previously described in detail [5]. Briefly, surgically resected astrocytoma samples were formalin-fixed and paraffinembedded prior to examination by a neuropathologist (CG) to determine grade III or grade IV status based on WHO criteria. Astrocytoma samples were arranged across two tissue independent microarrays in triplicate. One array contained 38 grade IV astrocytomas and the second 22 grade IV and 8 grade III astrocytomas. Fivemicron paraffin sections were cut for immunohistochemical localization of kallikreins. Patient demographics are provided in Additional file 1: Table S1, including age at surgery, gender and Eastern Cooperative Oncology Group (ECOG) performance score. Mayo Clinic Institutional Review Board approved the use of all human materials utilized in this study. Informed written consent was obtained prior to donation of tissue.

\section{Immunohistochemical analysis}

To determine the expression of kallikrein proteins in grade III and IV astrocytoma, tissue microarrays were immunostained using antibodies specific to KLK1, KLK6, KLK7, KLK8, KLK9, or KLK10 [14-16]. Deparaffinized sections were treated with $0.3 \%$ hydrogen peroxide in methanol, followed by rehydration in graded ethyl alcohols. Primary antibodies for each kallikrein were applied for $18 \mathrm{~h}$ at $4 \mathrm{C}$. KLK1 immunoreactivity was detected using a mouse monoclonal antibody M01H00003816 (Novus Biologicals, Littleton, CO). KLK6 was detected using a KLK6-specific monoclonal antibody (MSP-3-3) [14-16]. KLK7 [17, 18], KLK8 [19] and KLK10 [17] were detected with previously generated and validated rabbit polyclonal antibodies [20, 21]. KLK9 was detected using a rabbit polyclonal antibody PAB-10236 (Orbigen, San Diego, CA). Species appropriate biotinylated secondary antibodies (Jackson ImmunoResearch Laboratories, West Grove, PA) followed by peroxidaseconjugated streptavidin (Dako, Carpinteria, CA) and standard 3',3'-diaminobenzadine tetrahydrochloride immunohistochemistry were used to visualize kallikrein specific immunoreactivity. All immunostained tissue sections were counterstained with Gills hematoxylin.

All stained sections were imaged with a Bliss digital imaging system pairing an Axioplan microscope (Zeiss, Jena, Germany) with a slide scanner (Bacus Laboratories, Center Valley, PA). Two independent observers completed scoring without knowledge of tumor demographics. Scoring parameters consisted of the staining intensity (range 1-3: low, medium and high) and the percent tumor core stained (range 1-4: $25 \%$ increments). An IR score was calculated as the product of the staining intensity and percent stained. Scores from multiple cores from each tumor across the two observers were averaged [5]. Additional high-resolution images were prepared using a BX51 microscope (Olympus, Center Valley, PA) with a 100x-oil immersion objective and DP72 camera (Olympus).

\section{Statistical analysis}

Kallikrein staining parameters in grade III and IV astrocytoma were compared using the Kruskal-Wallis test. Cumulative survival probabilities were estimated using the Kaplan-Meier method. Cox proportional hazards regression was used to assess the association of kallikrein staining parameters with survival in the 
grade 4 astrocytomas. Both univariable and multivariable analyses were completed including age, gender and ECOG performance score as covariates. Analysis of Martingale residuals from the Cox proportional hazards regression models were used to assess the functional form of kallikrein parameters. Appropriate cut points for kallikrein parameters were determined based on this analysis (22). The cut points established were utilized to create survival curves using the KaplanMeier method. The dichotomized kallikrein parameters were then assessed using Cox proportional hazards regression as described above. In all analyses, $P<0.05$ was considered significant.

\section{Results}

\section{Kallikrein protein expression is increased in grade IV astrocytoma}

Expression of KLK1, KLK6, KLK7, KLK8, KLK9 and KLK10 protein was assessed by immunohistochemical analysis of grade III $(n=8)$ and grade IV $(n=60, n=55$ for KLK1) astrocytoma samples. The mean age was 48.1 (range 34-73) and 58.1 (range 33-84) for the grade III and grade IV patients, respectively. Grade III patients were $62.5 \%$ female and grade IV patients were $36.7 \%$ female. Additional demographic data regarding age, gender, extent of resection and ECOG performance scores are contained in Additional file 1: Table S1. All samples received a score for the intensity of the immunohistochemical staining, percent of the tissue core stained, and an IR score that is the product of the staining intensity and percent stained [5]. The range, median and distribution of scores are provided as box and whisker plots for each kallikrein by grade (Fig. 1). The most intense KLK-IR was seen in the grade IV tumors with antibodies recognizing KLK1, KLK6, KLK7 and KLK9. The intensity of immunoreactivity for KLK8 and KLK10 were low relative to the other kallikreins examined (see also Fig. 2).

To assess potential differences in kallikrein immunoreactivity across grade III and grade IV tumors, the mean scores for staining intensity, percent of tumor core stained, and IR scores were compared using the KruskalWallis test (Table 1). The mean intensity and IR scores were significantly higher in grade IV compared to grade III for all kallikreins examined (Table $1 ; P<0.03$, Kruskal-Wallis test). Also, the mean percent tumor core stained was significantly higher in grade IV relative to grade III for KLK6 (Table 1; $P=0.0005$, Kruskal-Wallis test). The mean percent tumor core stained was significantly higher in grade III compared to grade IV for KLK8 (Table 1; $P=0.0038$, Kruskal-Wallis test). The

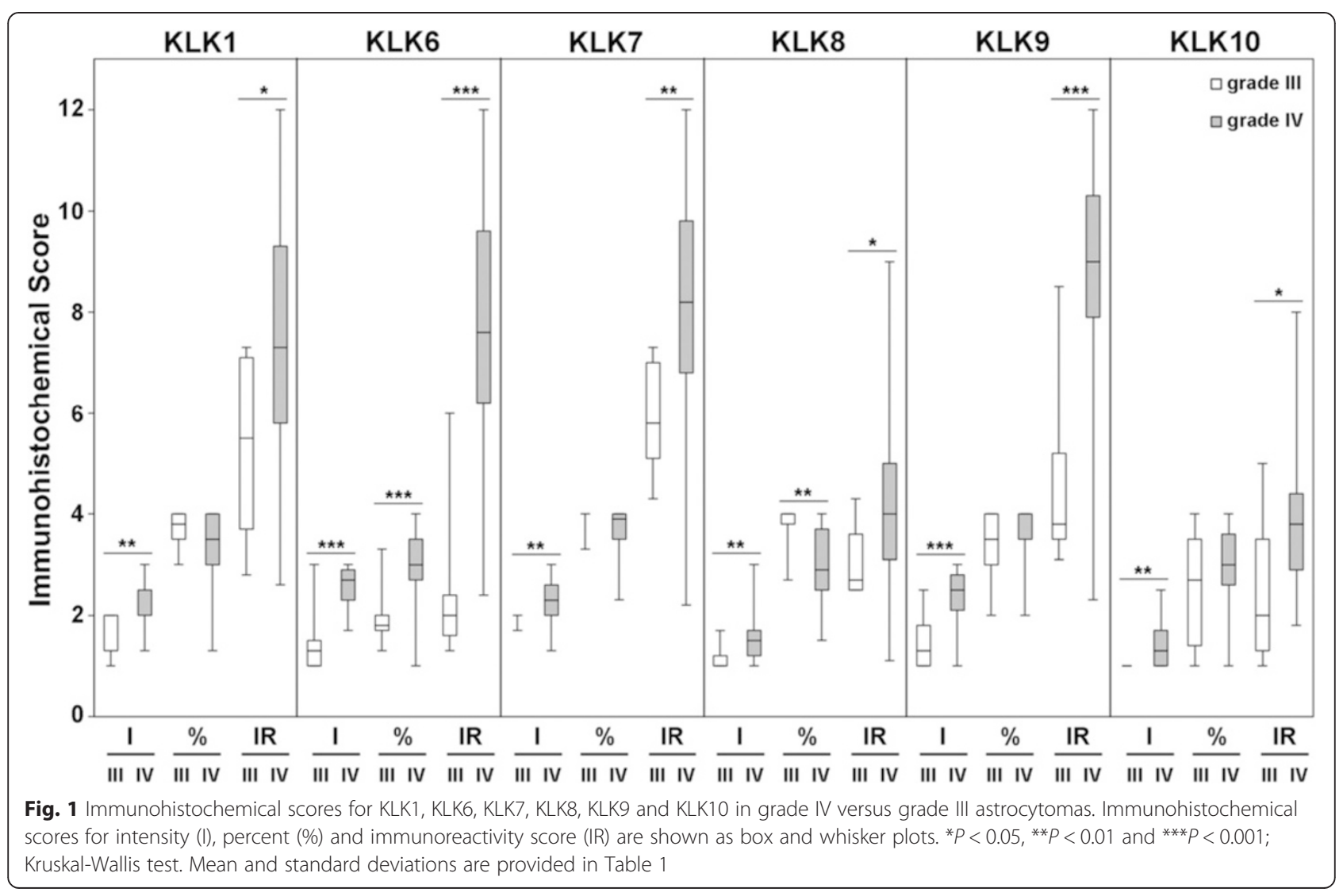




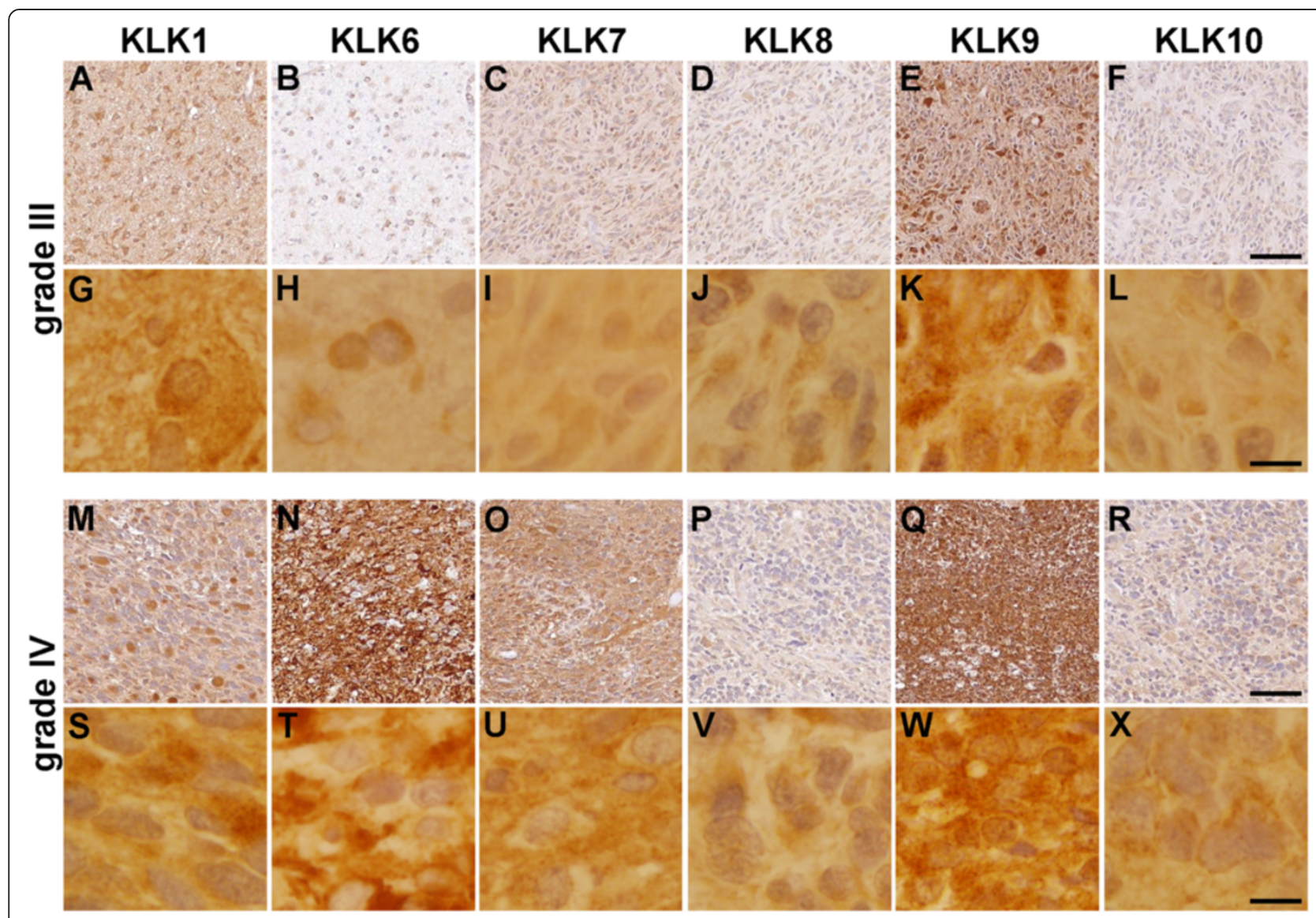

Fig. 2 Immunohistochemical staining for KLK1, KLK6, KLK7, KLK8, KLK9 and KLK10 in astrocytomas. Representative photomicrographs of grade III astrocytomas (a-I) and grade IV astrocytomas $(\mathbf{m}-\mathbf{x})$ stained for KLK1 $(\mathbf{a}, \mathbf{g}, \mathbf{m}$ and $\mathbf{s}), K L K 6(\mathbf{b}, \mathbf{h}, \mathbf{n}$ and $\mathbf{t}), K L K 7(\mathbf{c}, \mathbf{i}, \mathbf{o}$ and $\mathbf{u}), K L K 8(\mathbf{d}, \mathbf{j}, \mathbf{p}$ and $\mathbf{v}), K L K 9$ $(\mathbf{e}, \mathbf{k}, \mathbf{q}$ and $\mathbf{w})$, or KLK10 (f, I, $\mathbf{r}$ and $\mathbf{x})$. Images provided at low (a-f and $\mathbf{m}-\mathbf{r}$, scale bar $=50 \mu \mathrm{m})$ and high magnification $(\mathbf{g}-\mathbf{I}$ and $\mathbf{s}-\mathbf{x}$, scale bar $=10 \mu \mathrm{m}$ )

mean percent tumor core stained for KLK1, KLK7, KLK9 or KLK10 across grades III and IV were not significantly different.

The appearance of immunohistochemical staining for KLK1, KLK6, KLK7, KLK8, KLK9 and KLK10 in grade III and grade IV astrocytoma is shown in Fig. 2. As expected, immunoreactivity for each kallikrein was clearly visualized cytoplasmically. In addition, variable levels of extracellular staining were also apparent with the most intense extracellular staining seen in the case of KLK9, KLK7 and KLK6. In general, extracellular staining appeared the most intense in the higher grade gliomas (Fig. 2).

Table 1 Immunohistochemical scores for KLK1, KLK6, KLK7, KLK8, KLK9 and KLK10 in astrocytoma patients

\begin{tabular}{|c|c|c|c|c|c|c|c|c|c|}
\hline & \multicolumn{3}{|c|}{ Intensity \pm SD } & \multicolumn{3}{|c|}{ Percent \pm SD } & \multicolumn{3}{|l|}{$\mathrm{IR} \pm \mathrm{SD}$} \\
\hline & III & IV & $P^{a}$ & III & IV & $P^{a}$ & III & IV & $p^{a}$ \\
\hline KLK1 & $1.7 \pm 0.4$ & $2.2 \pm 0.4$ & $0.0063^{* *}$ & $3.7 \pm 0.4$ & $3.3 \pm 0.7$ & 0.096 & $5.4 \pm 1.8$ & $7.3 \pm 2.4$ & $0.031^{*}$ \\
\hline KLK6 & $1.5 \pm 0.7$ & $2.6 \pm 0.4$ & $0.0003^{* * *}$ & $2.0 \pm 0.6$ & $3.0 \pm 0.7$ & $0.0005^{* * *}$ & $2.4 \pm 1.5$ & $7.7 \pm 2.4$ & $<0.0001^{* * *}$ \\
\hline KLK7 & $2.0 \pm 0.1$ & $2.3 \pm 0.4$ & $0.0042^{* *}$ & $3.9 \pm 0.2$ & $3.7 \pm 0.4$ & 0.057 & $5.9 \pm 1.1$ & $8.2 \pm 2.1$ & $0.003^{* *}$ \\
\hline KLK8 & $1.1 \pm 0.2$ & $1.5 \pm 0.5$ & $0.0099^{* *}$ & $3.8 \pm 0.5$ & $3.0 \pm 0.8$ & $0.0038^{* *}$ & $3.0 \pm 0.7$ & $4.3 \pm 1.6$ & $0.022^{*}$ \\
\hline KLK9 & $1.4 \pm 0.6$ & $2.4 \pm 0.5$ & $0.0003^{* * *}$ & $3.4 \pm 0.7$ & $3.6 \pm 0.5$ & 0.42 & $4.6 \pm 1.8$ & $9.0 \pm 1.9$ & $0.0001^{* * *}$ \\
\hline KLK10 & $1.0 \pm 0.0$ & $1.4 \pm 0.4$ & $0.0012^{* *}$ & $2.5 \pm 1.2$ & $3.0 \pm 0.7$ & 0.36 & $2.4 \pm 1.4$ & $3.9 \pm 1.5$ & $0.011^{*}$ \\
\hline
\end{tabular}

Mean and standard deviation of KLK1, KLK6, KLK7, KLK8, KLK9 and KLK10 staining intensity, percent tumor core stained and immunoreactivity score across 60 grade IV astrocytoma specimens

${ }^{*} \mathrm{P}<0.05,{ }^{* *} \mathrm{P}<0.01$ and ${ }^{* * * P}<0.001$

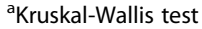




\section{Association of kallikrein immunoreactivity with survival in grade IV astrocytoma}

To determine the potential impact of differential kallikrein protein expression on patient survival, univariable Cox proportional hazards regression analysis was applied to correlate kallikrein staining with patient survival. Previous analysis of this patient cohort demonstrated that elevated KLK6-IR was associated with decreased patient survival (hazard ratio $[\mathrm{HR}]=1.16,95 \%$ confidence interval $[\mathrm{CI}]=1.02-1.31, P=0.02$; Table 2) [5]. The current analysis additionally demonstrated that elevated KLK7IR is associated with poor patient prognosis $(H R=1.17$, $\mathrm{CI}=1.02-1.34, P=0.03$; Table 2). Moreover, as the percent of the tumor core stained positive for KLK6 or KLK9 increased, survival decreased $(\mathrm{HR}=1.63, \mathrm{CI}=1.06-2.50$, $P=0.03$ and $\mathrm{HR}=2.25, \mathrm{CI}=1.21-4.22, P=0.01$, respectively; Table 2). After multivariable Cox modeling, utilizing gender, ECOG performance and age as covariates,

Table 2 Astrocytoma survival association with kallikrein protein expression

\begin{tabular}{|c|c|c|c|c|c|c|}
\hline & \multicolumn{3}{|c|}{ Univariable } & \multicolumn{3}{|c|}{ Adjusted $^{a}$} \\
\hline & $\mathrm{HR}$ & $95 \% \mathrm{Cl}$ & $P$ & $\mathrm{HR}$ & $95 \% \mathrm{Cl}$ & $P$ \\
\hline \multicolumn{7}{|l|}{ KLK1 } \\
\hline Intensity & 0.93 & $0.49-1.78$ & 0.83 & 1.10 & $0.54-2.23$ & 0.80 \\
\hline Percent & 1.21 & $0.81-1.80$ & 0.36 & 1.41 & $0.92-2.15$ & 0.11 \\
\hline $\mathbb{R}$ & 1.08 & $0.96-1.21$ & 0.21 & 1.13 & $0.99-1.29$ & 0.06 \\
\hline \multicolumn{7}{|l|}{ KLK6 } \\
\hline Intensity & 0.89 & $0.42-1.90$ & 0.77 & 0.73 & $0.32-1.68$ & 0.46 \\
\hline Percent & 1.63 & $1.06-2.50$ & $0.03^{*}$ & 1.35 & $0.87-2.10$ & 0.18 \\
\hline $\mathbb{R}$ & 1.16 & $1.02-1.31$ & $0.02^{*}$ & 1.10 & $0.96-1.25$ & 0.17 \\
\hline \multicolumn{7}{|l|}{ KLK7 } \\
\hline Intensity & 1.25 & $0.61-2.56$ & 0.54 & 0.95 & $0.45-2.03$ & 0.90 \\
\hline Percent & 1.96 & $0.91-4.22$ & 0.09 & 1.91 & $0.87-4.19$ & 0.11 \\
\hline $\mathbb{R}$ & 1.17 & $1.02-1.34$ & $0.03^{*}$ & 1.10 & $0.95-1.28$ & 0.20 \\
\hline \multicolumn{7}{|l|}{ KLK8 } \\
\hline Intensity & 0.87 & $0.46-1.66$ & 0.68 & 0.84 & $0.40-1.78$ & 0.65 \\
\hline Percent & 0.91 & $0.66-1.25$ & 0.54 & 0.96 & $0.68-1.36$ & 0.81 \\
\hline $\mathbb{R}$ & 0.98 & $0.83-1.16$ & 0.81 & 0.95 & $0.77-1.17$ & 0.61 \\
\hline \multicolumn{7}{|l|}{ KLK9 } \\
\hline Intensity & 0.65 & $0.38-1.13$ & 0.13 & 0.64 & $0.36-1.14$ & 0.13 \\
\hline Percent & 2.25 & $1.21-4.22$ & $0.01^{*}$ & 1.68 & $0.86-3.30$ & 0.13 \\
\hline IR & 1.05 & $0.90-1.22$ & 0.52 & 0.99 & $0.85-1.16$ & 0.90 \\
\hline \multicolumn{7}{|l|}{ KLK10 } \\
\hline Intensity & 1.14 & $0.59-2.20$ & 0.70 & 0.99 & $0.46-2.12$ & 0.97 \\
\hline Percent & 0.99 & $0.66-1.48$ & 0.94 & 1.10 & $0.72-1.69$ & 0.67 \\
\hline IR & 1.12 & $0.92-1.37$ & 0.27 & 1.03 & $0.82-1.28$ & 0.80 \\
\hline
\end{tabular}

Cox proportional hazards regression analysis assessing the association of kallikrein protein expression in 60 grade IV astrocytoma specimens. ${ }^{*} P \leq 0.03$, Cox proportional hazards regression analysis

${ }^{a}$ Adjusted for age, gender and performance score the association of kallikreins with survival was no longer significant. However, when only gender and ECOG performance scores were included as covariates in the multivariable analysis, scores for KLK6-IR and KLK9 \% tumor core stained, retained their significant association with survival $(\mathrm{HR}=1.15, \mathrm{CI}=1.01-1.30, P=0.03$ and $\mathrm{HR}=2.13, \mathrm{CI}=1.12-4.05, P=0.021$, respectively; Table 3).

Martingale residuals from the Cox proportional hazards regression model were used to assess the functional form of kallikrein parameters and when appropriate dichotomize the patients into groups. As we recently reported, patients with a KLK6-IR $<10(n=48)$ have a median survival of $408 \mathrm{~d}$, while patients with KLK6-IR $\geq 10$ $(n=12)$ have a significantly shorter survival time, $276 \mathrm{~d}$ $(\mathrm{HR}=2.36, \mathrm{CI}=1.19-4.68, P=0.014$; Table 3 and Fig. 3 ) [5]. This comparison remained significant when the data were adjusted for gender and ECOG performance scores $(\mathrm{HR}=2.4, \mathrm{CI}=1.20-4.78, P=0.01$; Table 3$)$. Patients with KLK6 \%<3 $(n=32)$ had a median survival of 419 d; patients with KLK6 \% $\geq 3(n=28)$ had a significantly shorter median survival of $320 \mathrm{~d}(\mathrm{HR}=2.07, \mathrm{CI}=1.14$ 3.76, $P=0.017$; Table 3 and Fig. 3). Multivariable analysis of KLK6 \% tumor core stained showed a significant correlation with survival, utilizing gender and ECOG as covariates $(\mathrm{HR}=1.93, \mathrm{CI}=1.03-3.63, P=0.04$; Table 3$)$. Patients with KLK7-IR $<9(n=38)$ had a median survival of 422 d; patients with KLK7-IR $\geq 9(n=22)$ had a significantly shorter median survival of $294 \mathrm{~d}(\mathrm{HR}=2.15$, $\mathrm{CI}=1.20-3.87, P=0.01$ ), which remained significantly different after adjustment for gender and performance score $(\mathrm{HR}=2.04, \quad \mathrm{CI}=1.10-3.77, \quad P=0.024)$. Patients with KLK9 \% tumor core stained < $4(n=29)$ had median survival of $422 \mathrm{~d}$; patients with KLK9 \% tumor core stained $=4(n=31)$ had a median survival of $311 \mathrm{~d}(\mathrm{HR}$ $=1.70, \mathrm{CI}=0.98-2.95, P=0.061$; Table 3 and Fig. 3 ).

\section{Discussion}

Through a comprehensive parallel analysis of immunoreactivity for six kallikreins in grade III and IV astrocytoma we determined that tumor core staining for KLK7 and KLK9, like KLK6, has significant prognostic value with regard to patient survival. Specifically, higher tumor core levels of KLK6, KLK7 and KLK9 were each associated with reduced GBM patient survival. These findings highlight the likely significance of each of these secreted serine proteases to the pathophysiology of high-grade glioma and the need for additional studies to define the mechanism of action, significance to therapy resistance and their potential utility as therapeutic targets to improve patient outcomes.

Prior studies demonstrate that higher levels of KLK6 immunoreactivity in grade IV astrocytoma are associated with poor patient survival [5]. Expression of KLK6 RNA 
Table 3 Survival analysis of immunohistochemical scores after cut point analysis

\begin{tabular}{|c|c|c|c|c|c|c|c|c|}
\hline & \multirow[b]{2}{*}{$N$} & \multirow[b]{2}{*}{ Median Survival } & \multicolumn{3}{|c|}{ Univariable Analysis } & \multicolumn{3}{|c|}{ Multivariable Analysis } \\
\hline & & & Hazard Ratio & $95 \% \mathrm{Cl}$ & $P^{a}$ & Hazard Ratio & $95 \% \mathrm{Cl}$ & $P^{a}$ \\
\hline \multirow[t]{2}{*}{ KLK6 \% } & 60 & 355 & 1.63 & $1.06-2.50$ & 0.027 & 1.35 & $0.87-2.10$ & $0.18^{\mathrm{b}}$ \\
\hline & & & & & & 1.55 & $1.10-2.40$ & $0.052^{c}$ \\
\hline$<3$ & 32 & 419 & & & & & & \\
\hline \multirow[t]{2}{*}{$\geq 3$} & 28 & 320 & 2.07 & $1.14-3.76$ & 0.017 & 1.36 & $0.72-2.57$ & $0.34^{\mathrm{b}}$ \\
\hline & & & & & & 1.93 & $1.03-3.63$ & $0.04^{c}$ \\
\hline \multirow[t]{2}{*}{ KLK6-IR } & 60 & 355 & 1.16 & $1.02-1.31$ & 0.020 & 1.10 & $0.96-1.25$ & $0.17^{b}$ \\
\hline & & & & & & 1.15 & $1.01-1.30$ & $0.03^{c}$ \\
\hline$<10$ & 48 & 408 & & & & & & \\
\hline \multirow[t]{2}{*}{$\geq 10$} & 12 & 276 & 2.36 & $1.19-4.68$ & 0.014 & 1.72 & $0.84-3.54$ & $0.14^{\mathrm{b}}$ \\
\hline & & & & & & 2.4 & $1.20-4.78$ & $0.01^{c}$ \\
\hline \multirow[t]{2}{*}{ KLK7-IR } & 60 & 355 & 1.17 & $1.02-1.34$ & 0.025 & 1.10 & $0.95-1.28$ & $0.20^{b}$ \\
\hline & & & & & & 1.15 & $1.00-1.32$ & $0.057^{c}$ \\
\hline$<9$ & 38 & 422 & & & & & & \\
\hline \multirow[t]{2}{*}{$\geq 9$} & 22 & 294 & 2.15 & $1.20-3.87$ & 0.010 & 1.47 & $0.77-2.80$ & $0.24^{b}$ \\
\hline & & & & & & 2.04 & $1.10-3.77$ & $0.024^{c}$ \\
\hline \multirow[t]{2}{*}{ KLK9 \% } & 60 & 355 & 2.25 & $1.21-4.22$ & 0.011 & 1.68 & $0.86-3.3$ & $0.13^{b}$ \\
\hline & & & & & & 2.13 & $1.12-4.05$ & $0.021^{c}$ \\
\hline$<4$ & 29 & 422 & & & & & & \\
\hline \multirow[t]{2}{*}{$=4$} & 31 & 311 & 1.70 & $0.98-2.95$ & 0.061 & 1.42 & $0.79-2.57$ & $0.24^{\mathrm{b}}$ \\
\hline & & & & & & 1.58 & $0.89-2.79$ & $0.12^{c}$ \\
\hline
\end{tabular}

Cox proportional hazards regression analysis assessing the association of kallikrein protein expression with patient survival in 60 grade IV astrocytoma specimens aproportional hazards regression

${ }^{\mathrm{b}}$ Adjusted for age, gender and performance score

${ }^{\mathrm{c}}$ Adjusted for gender and performance score

is also linked to poor patient survival in a group of intracranial malignancies, including glioblastomas, meningiomas, oligodendrogliomas, ependymomas and other rare malignancies and brain metastases [12]. KLK6 expression is associated with an unfavorable prognosis in ovarian [3] and colorectal cancers [22]. In contrast, KLK6 expression in breast cancer appears to have a protective role, with elevated expression being linked to reduced proliferation and tumorigenicity. Expression of KLK6 is also reduced in metastatic lymph nodes [23].

Paralleling what we have reported for KLK6 [5], results herein suggest that elevated levels of KLK7 expression are associated with poor patient survival. Supporting this, a prior study examining a mixed cohort of intracranial malignancies similarly demonstrated that elevations in KLK7 expression are associated with poor patient survival [13]. Both studies support the idea that KLK7 expression leads to more aggressive brain tumors. KLK7 is also associated with reduced survival times in ovarian [24], breast [25] and colon cancer [26]. By contrast, the current study and prior efforts suggest that changes in KLK8 expression in GBM or other intracranial malignancies have little association with prognosis [13]. In head and neck squamous cell carcinoma, KLK8 expression is down regulated in metastases, but it is not associated with improved survival [27]. Pointing to potential cancer-type specific effects as described for KLK6, KLK8 is a favorable prognostic indicator in ovarian cancer [28], but an unfavorable indicator in lung cancer [29].

The current study provides the first evidence that KLK9 protein expression may be associated with poor prognosis in glioma patients. Again pointing to potential tumor specific effects of kallikreins, KLK9 was previously found to be expressed at higher levels in low grade breast and ovarian cancers [30, 31]. In breast cancer, KLK9 expression was also higher in patients with smaller tumors and was associated with increased patient survival, particularly patients that are estrogen and progesterone receptor negative [31]. In the case of ovarian cancer, KLK9 expression is a predictor of longer overall survival in patients with lower grade tumors [30]. Specific regulatory patterns of kallikreins across cancers may be related to hormone regulation, as proposed for KLK9 in both breast and ovarian cancer. Interestingly, KLK10 is a proposed tumor suppressor gene in breast carcinoma cell lines, blocking tumorigenicity in an 


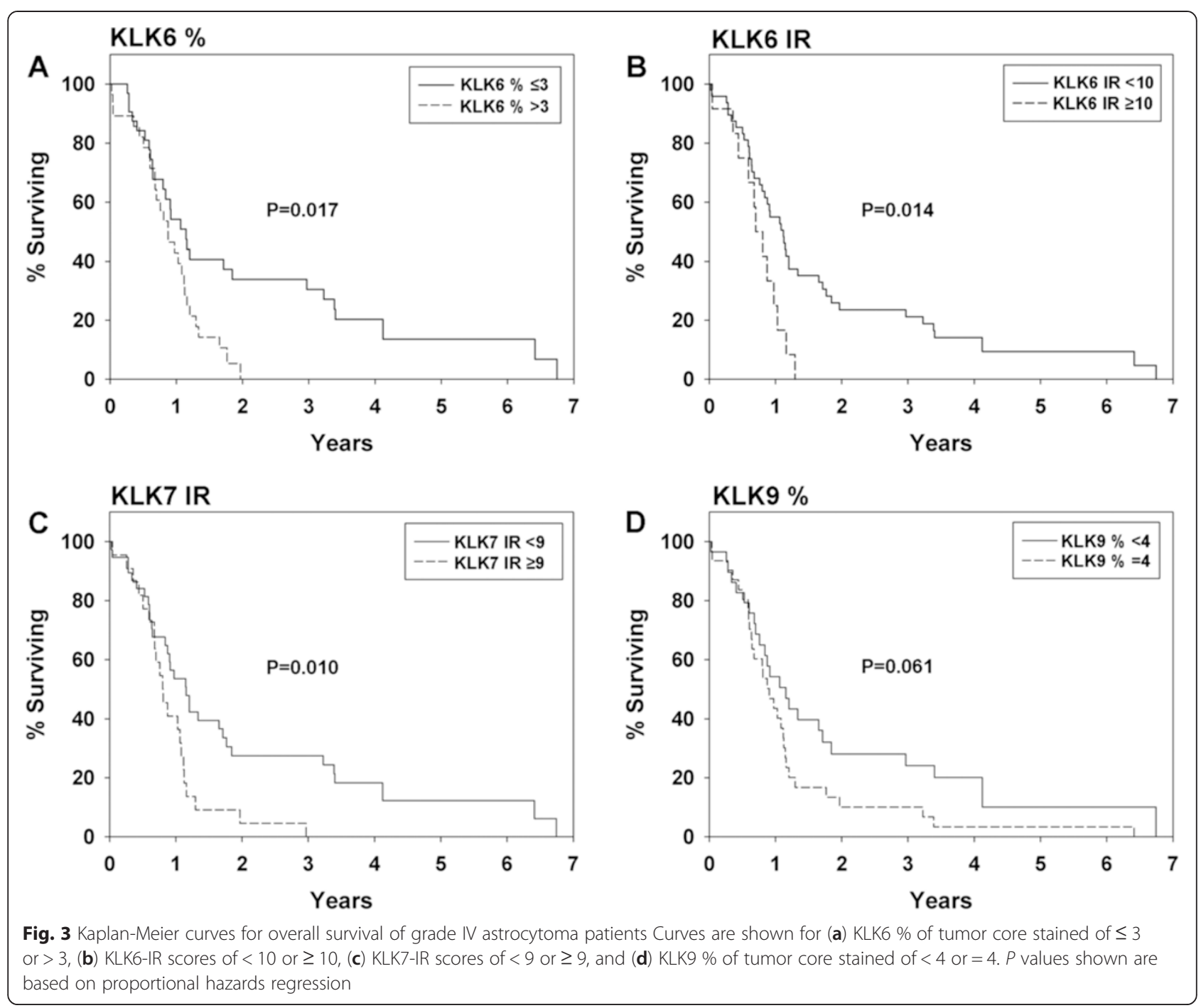

in vivo breast cancer model [32]. However, underscoring the importance of considering tumor-type specific effects, KLK10 has been found to be an unfavorable prognostic indicator in gastric, colorectal and ovarian cancer [33-35]. Here we report that KLK10 expression is increased with astrocytoma grade, but no association with patient survival was observed.

Our previous studies demonstrate that KLK6 over expression promotes resistance of GBM cell lines to cell death inducing agents, including radiation and temozolomide, the current standard of GBM patient care [5]. Interestingly, the KLK6-mediated activation of PAR1 was shown to play an essential role in its ability to promote glioma cell survival [5]. Notably, KLK6 also promotes survival of the Jurkat leukemia $\mathrm{T}$ cell line in a PAR1 dependent fashion [36]. In melanoma cells, PAR1 is activated by KLK6 promoting intracellular calcium flux and tumor cell invasion [37]. Together these studies support the concept that KLK6 mediates its effects in astrocytoma cells in a PAR1-dependent fashion and that this is likely to involve both cell survival and invasion.

KLK7 overexpression increases cell invasion in glioma cell lines in vitro matrigel assay [13]. Moreover, overexpression of KLK7 in colon cancer cell lines promotes proliferation and tumorigenicity [38]. The signaling pathways participating in KLK7-mediated effects in tumor cells have not been elucidated, but effects on cell invasion and proliferation may account for the shortened survival times we observe in GBM patients with elevated tumor KLK7 expression.

The current studies are among the first to examine KLK9 in malignancy and results of interest since elevations in tumor KLK9 were found to be associated with higher grade gliomas. These findings point to the need for additional studies to determine the biological effects of kallikreins in glioma malignancy and whether these effects are mediated by PAR-dependent and/or independent actions, such as extracellular matrix turnover. In addition to 
direct effects on tumor cell behavior, it is also possible that the kallikreins identified herein participate in activation, or inactivation, cascades with other kallikreins or enzymes involved in the fibrinolytic or thrombolytic systems [21].

\section{Conclusion}

Data presented here demonstrate that elevated levels of KLK6, KLK7 and KLK9 proteins are associated with poor GBM patient survival. Our prior studies suggest that KLK6 directly promotes glioma cell survival, including resistance to radiation and temozolomide, in a PAR1-dependent manner. The current work therefore points to the need for additional studies to determine the potential pathophysiological roles of KLK7 and KLK9 in glioma malignancy and any parallel involvement of PAR-activation in mediating their effects. This knowledge will be key to potential future studies in which kallikreins or the receptors they activate could be targeted therapeutically to improve patient survival. Importantly, the current results suggest future studies to determine any impact of elevated kallikrein levels on therapy responsiveness. Finally, analysis of the mechanisms by which kallikreins are elevated in GBM, albeit by gene duplications, hormonal regulation, epigenetic changes, or other means, will be of interest and potentially important to understanding the differential expression and outcomes these novel serine proteases exert across a wide range of malignancies.

\section{Additional files}

Additional file 1: Table S1. Patient demographic data. (DOCX $40 \mathrm{~kb}$ )

\section{Abbreviations}

KLK: Kallikrein; IR: Immunoreactivity score; PSA: Prostate specific antigen; GBM: Glioblastoma multiforme; CNS: Central nervous system; WHO: World Health Organization; ECOG: Eastern Cooperative Oncology Group; HR: Hazard ratio; $\mathrm{Cl}$ : Confidence interval.

\section{Competing interests}

The authors declare that they have no competing interests.

\section{Authors' contributions}

KD carried out data analysis and participated in figure preparation and drafted the manuscript. CG designed and constructed the tissue microarrays, as well as performed the histologic review of all tissue samples included in the microarray. PAD performed the statistical analysis and participated in the table preparation. EPD was responsible for the production of essential antibodies. IAS conceived and designed the study, performed the immunoassays and data analysis, and helped to draft the manuscript. All authors read and approved of the final manuscript.

\section{Acknowledgments}

National Institutes of Health Brain Tumor SPORE grant career development award P50CA108961 (I.A.S), the National Institutes of Health Mayo Neuro-oncology Training Grant (T32 NS07494, K.L.D), National Institutes of Neurological Disorders and Stroke R01NS052471 (I.A.S), and an Accelerated Regenerative Medicine Award from the Center for Regenerative Medicine, Mayo Clinic (I.A.S.)

\section{Author details}

'Department of Physical Medicine and Rehabilitation, Mayo Medical and Graduate School, Mayo Clinic Rochester, 200 First Street SW, Rochester, MN 55905, USA. ${ }^{2}$ Department of Laboratory Medicine and Pathology, Mayo Medical and Graduate School, Mayo Clinic Rochester, 200 First Street SW, Rochester, MN 55905, USA. ${ }^{3}$ Biomedical Statistics and Informatics, Mayo Medical and Graduate School, Mayo Clinic Rochester, 200 First Street SW, Rochester, MN 55905, USA. ${ }^{4}$ Department of Pathology and Laboratory Medicine, Mount Sinai Hospital, 600 University Ave, Toronto, ON M5T 3 L9, Canada. ${ }^{5}$ Department of Physiology and Biomedical Engineering, Mayo Medical and Graduate School, Mayo Clinic Rochester, 200 First Street SW, Rochester, MN 55905, USA.

Received: 9 January 2015 Accepted: 16 July 2015

Published online: 01 August 2015

\section{References}

1. Emami N, Diamandis EP. Utility of kallikrein-related peptidases (KLKs) as cancer biomarkers. Clin Chem. 2008;54(10):1600-7.

2. Paliouras M, Diamandis EP. The kallikrein world: an update on the human tissue kallikreins. Biol Chem. 2006;387(6):643-52.

3. Bayani J, Marrano P, Graham C, Zheng Y, Li L, Katsaros D, et al. Genomic instability and copy-number heterogeneity of chromosome $19 q$, including the kallikrein locus, in ovarian carcinomas. Mol Oncol. 2011;5(1):48-60.

4. Zhu X, Albertsen PC, Andriole GL, Roobol MJ, Schroder FH, Vickers AJ. Risk-based prostate cancer screening. Eur Urol. 2012;61(4):652-61.

5. Drucker KL, Paulsen AR, Giannini C, Decker PA, Blaber SI, Blaber M, et al. Clinical significance and novel mechanism of action of kallikrein 6 in glioblastoma. Neuro Oncol. 2013;15(3):305-18.

6. Smith JS, Alderete B, Minn Y, Borell TJ, Perry A, Mohapatra G, et al. Localization of common deletion regions on $1 p$ and $19 q$ in human gliomas and their association with histological subtype. Oncogene. 1999;18(28):4144-52.

7. Nakamura M, Yang F, Fujisawa H, Yonekawa Y, Kleihues P, Ohgaki H. Loss of heterozygosity on chromosome 19 in secondary glioblastomas. J Neuropathol Exp Neurol. 2000;59(6):539-43.

8. Jenkins RB, Blair H, Ballman KV, Giannini C, Arusell RM, Law M, et al. A $\mathrm{t}(1 ; 19)(q 10 ; p 10)$ mediates the combined deletions of $1 p$ and $19 q$ and predicts a better prognosis of patients with oligodendroglioma. Cancer Res. 2006;66(20):9852-61.

9. Smith JS, Tachibana I, Lee HK, Qian J, Pohl U, Mohrenweiser HW, et al. Mapping of the chromosome 19 q-arm glioma tumor suppressor gene using fluorescence in situ hybridization and novel microsatellite markers. Genes Chromosomes Cancer. 2000;29(1):16-25.

10. Korshunov A, Sycheva R, Golanov A. The prognostic relevance of molecular alterations in glioblastomas for patients age $<50$ years. Cancer. 2005;104(4):825-32.

11. Huhn SL, Mohapatra G, Bollen A, Lamborn K, Prados MD, Feuerstein BG. Chromosomal abnormalities in glioblastoma multiforme by comparative genomic hybridization: correlation with radiation treatment outcome. Clin Cancer Res. 1999;5(6):1435-43.

12. Talieri M, Zoma M, Devetzi M, Scorilas A, Ardavanis A. Kallikrein-related peptidase 6 (KLK6) gene expression in intracranial tumors. Tumour Biol. 2012;33(5):1375-83.

13. Prezas P, Scorilas A, Yfanti C, Viktorov P, Agnanti N, Diamandis E, et al. The role of human tissue kallikreins 7 and 8 in intracranial malignancies. Biol Chem. 2006;387(12):1607-12.

14. Scarisbrick IA, Blaber SI, Lucchinetti CF, Genain CP, Blaber M, Rodriguez M. Activity of a newly identified serine protease in CNS demyelination. Brain. 2002;125(Pt 6):1283-96

15. Scarisbrick IA, Asakura K, Blaber S, Blaber M, Isackson PJ, Bieto T, et al. Preferential expression of myelencephalon-specific protease by oligodendrocytes of the adult rat spinal cord white matter. Glia. 2000;30(3):219-30.

16. Scarisbrick IA, Isackson PJ, Ciric B, Windebank AJ, Rodriguez M. MSP, a trypsin-like serine protease, is abundantly expressed in the human nervous system. J Comp Neurol. 2001;431(3):347-61.

17. Diamandis EP, Scorilas A, Kishi T, Blennow K, Luo LY, Soosaipillai A, et al. Altered kallikrein 7 and 10 concentrations in cerebrospinal fluid of patients 
with Alzheimer's disease and frontotemporal dementia. Clin Biochem. 2004:37(3):230-7.

18. Petraki CD, Papanastasiou PA, Karavana VN, Diamandis EP. Cellular distribution of human tissue kallikreins: immunohistochemical localization. Biol Chem. 2006;387(6):653-63.

19. Komatsu N, Suga Y, Saijoh K, Liu AC, Khan S, Mizuno Y, et al. Elevated human tissue kallikrein levels in the stratum corneum and serum of peeling skin syndrome-type B patients suggests an over-desquamation of corneocytes. J Invest Dermatol. 2006;126(10):2338-42.

20. Shaw JL, Diamandis EP. Distribution of 15 human kallikreins in tissues and biological fluids. Clin Chem. 2007;53(8):1423-32.

21. Radulovic M, Yoon H, Larson N, Wu J, Linbo R, Burda JE, et al. Kallikrein cascades in traumatic spinal cord injury: in vitro evidence for roles in axonopathy and neuron degeneration. J Neuropathol Exp Neurol. 2013;72(11):1072-89.

22. Christodoulou S, Alexopoulou DK, Kontos CK, Scorilas A, Papadopoulos IN. Kallikrein-related peptidase-6 (KLK6) mRNA expression is an independent prognostic tissue biomarker of poor disease-free and overall survival in colorectal adenocarcinoma. Tumour Biol. 2014;35(5):4673-85.

23. Pampalakis G, Prosnikli E, Agalioti T, Vlahou A, Zoumpourlis V, Sotiropoulou G. A tumor-protective role for human kallikrein-related peptidase 6 in breast cancer mediated by inhibition of epithelial-to-mesenchymal transition. Cancer Res. 2009;69(9):3779-87.

24. Kyriakopoulou LG, Yousef GM, Scorilas A, Katsaros D, Massobrio M, Fracchioli $\mathrm{S}$, et al. Prognostic value of quantitatively assessed KLK7 expression in ovarian cancer. Clin Biochem. 2003;36(2):135-43.

25. Talieri M, Diamandis EP, Gourgiotis D, Mathioudaki K, Scorilas A. Expression analysis of the human kallikrein 7 (KLK7) in breast tumors: a new potential biomarker for prognosis of breast carcinoma. Thromb Haemost. 2004;91(1):180-6.

26. Talieri M, Mathioudaki K, Prezas P, Alexopoulou DK, Diamandis EP, Xynopoulos D, et al. Clinical significance of kallikrein-related peptidase 7 (KLK7) in colorectal cancer. Thromb Haemost. 2009;101(4):741-7.

27. Liu CJ, Liu TY, Kuo LT, Cheng HW, Chu TH, Chang KW, et al. Differential gene expression signature between primary and metastatic head and neck squamous cell carcinoma. J Pathol. 2008;214(4):489-97.

28. Magklara A, Scorilas A, Katsaros D, Massobrio M, Yousef GM, Fracchioli S, et al. The human KLK8 (neuropsin/ovasin) gene: identification of two novel splice variants and its prognostic value in ovarian cancer. Clin Cancer Res. 2001;7(4):806-11.

29. Planque C, Choi YH, Guyetant S, Heuze-Vourc'h N, Briollais L, Courty Y. Alternative splicing variant of kallikrein-related peptidase 8 as an independent predictor of unfavorable prognosis in lung cancer. Clin Chem. 2010;56(6):987-97.

30. Yousef GM, Kyriakopoulou LG, Scorilas A, Fracchioli S, Ghiringhello B, Zarghooni M, et al. Quantitative expression of the human kallikrein gene 9 (KLK9) in ovarian cancer: a new independent and favorable prognostic marker. Cancer Res. 2001;61(21):7811-8.

31. Yousef GM, Scorilas A, Nakamura T, Ellatif MA, Ponzone R, Biglia N, et al. The prognostic value of the human kallikrein gene 9 (KLK9) in breast cancer. Breast Cancer Res Treat. 2003;78(2):149-58.

32. Goyal J, Smith KM, Cowan JM, Wazer DE, Lee SW, Band V. The role for NES1 serine protease as a novel tumor suppressor. Cancer Res. 1998;58(21):4782-6.

33. Kolin DL, Sy K, Rotondo F, Bassily MN, Kovacs K, Brezden-Masley C, et al. Prognostic significance of human tissue kallikrein-related peptidases 6 and 10 in gastric cancer. Biol Chem. 2014;395(9):1087-93.

34. Alexopoulou DK, Papadopoulos IN, Scorilas A. Clinical significance of kallikrein-related peptidase (KLK10) mRNA expression in colorectal cancer. Clin Biochem. 2013;46(15):1453-61.

35. Luo LY, Katsaros D, Scorilas A, Fracchioli S, Piccinno R, Rigault de la Longrais IA, et al. Prognostic value of human kallikrein 10 expression in epithelial ovarian carcinoma. Clin Cancer Res. 2001;7(8):2372-9.

36. Scarisbrick IA, Epstein B, Cloud BA, Yoon H, Wu J, Renner DN, et al. Functional role of kallikrein 6 in regulating immune cell survival. PLoS One. 2011;6(3), e18376.

37. Krenzer S, Peterziel H, Mauch C, Blaber SI, Blaber M, Angel P, et al. Expression and function of the kallikrein-related peptidase 6 in the human melanoma microenvironment. J Invest Dermatol. 2011;131(11):2281-8.
38. Walker F, Nicole P, Jallane A, Soosaipillai A, Mosbach V, Oikonomopoulou K, et al. Kallikrein-related peptidase 7 (KLK7) is a proliferative factor that is aberrantly expressed in human colon cancer. Biol Chem. 2014;395(9):1075-86.

\section{Submit your next manuscript to BioMed Central and take full advantage of:}

- Convenient online submission

- Thorough peer review

- No space constraints or color figure charges

- Immediate publication on acceptance

- Inclusion in PubMed, CAS, Scopus and Google Scholar

- Research which is freely available for redistribution 